\title{
Psychotherapy of phantom pain in oncology
}

\begin{abstract}
Psychotherapy using the "mirror box" (by V. Ramachandran) and progressive muscle relaxation (by E. Jacobson) in combination with music are effective in correcting phantom pain in patients with malignant tumors of bones and soft tissues. Analysis of VAS data before and after the correction showed a significant reduction in phantom pain in the patients examined: from $73.9+9.0$ to $58.9 \pm 7.9$ scores, respectively (Student's t-test $=4.7 ; \mathrm{P}<0.05$ ). By Wong-Baker scale, a significant decrease in the intensity of phantom pain from $7.7 \pm 1.1$ to $5.7 \pm 1.1$ scores (Student's t-test $=5, \mathrm{P}<0.05$ ).
\end{abstract}

Keywords: psychotherapy, phantom pain, VAS, Wong-Baker scale, method of "mirror therapy", progressive muscular relaxation
Volume 9 Issue 6 - 2018

Tkachenko G, Stepanova A

N.N. Blokhin NMRC of Oncology of the Ministry of Health of Russia, Russia

Correspondence: Tkachenko G, N.N. Blokhin, NMRC of Oncology of the Ministry of Health of Russia, Moscow, Russia, Emailmifg7।@mail.ru

Received: April 20, 2018| Published: November 20, 2018

\section{Introduction}

Amputation or removal of any part is usually associated with a feeling that the missing part is still present and causes disorders of different types of sensitivity, but mainly excruciating pain sensations. The phantom pain is a false sensation, an illusion of the presence of the missing part of the body. The symptoms of phantom pain are especially pronounced and often manifest themselves after surgery for malignant tumors. According to different authors, the PP manifestations in cancer patients are observed with a high frequency from 30 to $90 \%{ }^{1}$ Phantom pains can appear immediately after surgery but their onset can be observed after a considerable time after amputation. With time, the pain becomes less pronounced, and its incidence decreases. However, there are cases when phantom pain persists even 5-7 years after amputation. As clinical experience shows, even with the complex application of modern anti-neuropathic agents, opioid and non-opioid analgesics, regional blocks, the treatment of phantom pain in many cases does not give positive results. ${ }^{2,3}$ That is why it is important to search for effective methods of prophylaxis and correction of phantom pain in cancer patients. Along with medical treatment, in many countries the great attention has been recently paid to the psychotherapy. It has been proven that one of the valuable tools that can be used to reduce the intensity of pain is psychological interventions and various methods of psychotherapy, which, on the one hand, are safe, but, on the other hand, are very effective. ${ }^{1,4}$ In the international practice, hypnotherapy, biological feedback, "mirror box" technique, autogenic training, neuroinduction and self-induced hypnosis, and many other methods are widely used. ${ }^{1,5}$ In this regard, the studies in this area are relevant as well as the use not only of existing techniques, but the creation of new effective methods of psychological correction of phantom pain.

\section{Purpose}

Evaluation of the effectiveness of psychotherapy methods in correcting phantom pain in patients with malignant tumors of bones and soft tissues.

\section{Material and methods}

14 patients with malignant tumors of bones and soft tissues were included in the study: bone osteosarcoma (35.7\%), soft tissue sarcoma $(64.3 \%)$, who underwent amputations and exarticulation of extremities in combination with lymphadenectomy aged 36 to 60 years. Clinical and psychological research provided for the use of questionnaires to assess the intensity of pain: visual analogue scale (VAS) and Wong-Baker faces pain scale. Statistical processing of the results was carried out using a package of statistical programs. The comparative analysis was carried out using Student's t-test. The significance of differences in indices was $P<0.05$. We have chosen the methods that proved to be the simplest but at the same time, effective for reducing phantom pains: "mirror box" technique and progressive muscle relaxation. 10 sessions of "mirror therapy" (by V. Ramachandran) and 10 sessions of progressive muscular relaxation (by E. Jacobson) have been conducted with each patient, individually, in combination with music or sounds of nature. The sessions were held 2 times a day for 30 minutes of "mirror therapy" and 45 minutes of relaxation. The number of sessions was determined by the terms of the patient's hospitalization after the surgery (10-14 days).

The proposed method included four steps:

1. Stage I - before surgery - psychological preparation of the patient for amputation (exarticulation and other surgical interventions). At this stage, information and explanatory work was carried out with the patients, the content of which was determined by the specifics of the diseases: the patient received information from the doctor about the forthcoming surgical intervention and its consequences (including the possible development of phantom pain).

2. Stage II - after surgery - psychological diagnosis: Before the psychotherapy, a psychodiagnosis was carried out, including an assessment of the pain syndrome, as well as the actual mental state for detecting depression, high level of anxiety caused by oncological disease and greatly complicating the correction of phantom pain. Carrying out psychodiagnosis is important for increasing the patient's motivation for psychotherapy.

3. Stage III - psychotherapy - conducting psychotherapeutic methods aimed at reducing the pain.

4. Stage IV - (before discharge) - repeated psychodiagnosis to evaluate the effectiveness of the therapy.

\section{Results and discussion}

Quantitative analysis of VAS data before and after the correction showed a significant reduction in phantom pain in the patients 
examined: from $73.9+9.0$ to $58.9 \pm 7.9$ scores, respectively (Student's $\mathrm{t}$-test $=4.7 ; P<0.05$ ). There was a clear improvement in the overall well-being of the patients after the course of correction. A qualitative analysis of the test results showed that phantom pains of varying intensity were initially observed in all patients: only 1 patient (7.1\%) showed moderate pain; 1 patient noted unbearable pain (7.1\%); severe pain was noted in all others $(85.8 \%)$. After the course of psychological correction, the number of patients with moderate to severe pain significantly increased to $78.6 \%$ and the number of patients with a pronounced level of phantom pains decreased (21.4\%). By WongBaker scale, a significant decrease in the intensity of phantom pain from $7.7 \pm 1.1$ to $5.7 \pm 1.1$ scores (Student's t-test $=5, P<0.05$ ). The intensity of pain of 8 scores was revealed in 2 patients $(14.3 \%)$, of 4 scores - in 1 patient (7.1\%), of 6 scores - in 11 patients $(78.6 \%)$. Along with a decrease in phantom pain, all patients demonstrated improvement in sleep, mood, and increased physical activity.

\section{Conclusion}

The study showed that the proposed methods of psychotherapy using the "mirror box" and progressive muscle relaxation in combination with music are effective in correcting phantom pain in patients with malignant tumors of bones and soft tissues.

\section{Acknowledgements}

None.

\section{Conflict of interest}

The author declares that there is no conflict of interest.

\section{References}

1. Cárdenas K, Aranda M. Psychotherapies for the Treatment of Phantom Limb Pain. Rev Colomb Psiquiatr. 2017;46(3):178-186.

2. Reuben S. Persistent postoperative pain: pharmacological treatment strategies in the postoperative setting, Pain 2008 - an Updated Review. Castro-Lopes J, Raja S, Scmelz M, editors. IASP Press: Seattle;2008;159-169.

3. Sindrup SH. Clinical pharmacology of antiepileptics and antidepressants in the management of neuropathic pain. Pain 2008- an Updated Review. Castro-Lopes J, Raja S, Schmelz M, editors. JASP Press. Seattle;2008:217-223.

4. Castelnuovo G, Giusti EM, Manzoni GM., et al. Psychological Treatments and Psychotherapies in the Neurorehabilitation of Pain: Evidences and Recommendations from the Italian Consensus Conference on Pain in Neurorehabilitation. Front Psychol. 2016; 19;7:115.

5. Diers M, Flor H. Phantom limb pain. Psychological treatment strategies. Schmerz. 2013;27(2):205-213. 